\title{
Integrand reduction at NLO and beyond
}

\author{
Hans van Deurzen, Gionata Luisoni, Edoardo Mirabella, Tiziano Peraro*, \\ Ulrich Schubert \\ Max-Planck Insitut für Physik, München, Germany \\ E-mail: \{hdeurzen, luisonig, mirabell, peraro, schubert\}@mpp.mpg.de
}

\section{Pierpaolo Mastrolia}

Max-Planck Insitut für Physik, München, Germany

Dipartimento di Fisica e Astronomia, Università di Padova, and INFN Sezione di Padova, Italy

E-mail: ppaolo@mpp.mpg • de

\section{Giovanni Ossola}

Physics Department, New York City College of Technology, The City University of New York, USA

The Graduate School and University Center, The City University of New York, USA

E-mail: gossolaecitytech. cuny .edu

\begin{abstract}
The integrand-level methods for the reduction of scattering amplitudes are powerful techniques for the analysis and the computation of loop integrals, which have already been successfully applied and automated at one-loop. Moreover, some very interesting progress has recently been made towards the higher-loop extension of such techniques. In this presentation, we review the basics principles of integrand-reduction methods within a coherent framework we developed, which can be applied to any integrand at any number of loops and is based on simple concepts of algebraic geometry, such as multivariate polynomial division. We particularly focus on semianalytic and algebraic techniques, such as the Laurent series expansion which we exploited to improve the one-loop reduction with the library NINJA, and the multi-loop divide-and-conquer approach which can always be used to find the integrand decomposition of any Feynman graph in a finite number of algebraic operations.
\end{abstract}

The European Physical Society Conference on High Energy Physics

18-24 July, 2013

Stockholm, Sweden

\footnotetext{
*Speaker.
} 


\section{Overview}

Scattering amplitudes are analytic functions of the momenta of the particles involved and they can be studied by exploring their singularity structure [1,2]. The integrand reduction methods, originally developed for one-loop diagrams $[3,4]$ and more recently extended to higher loops [5-9], use the singularity structure of the integrands to decompose the (integrated) amplitudes in terms of Master Integrals (MI's). They rely on the existence of a relation between the numerator and the denominators of each Feynman integral. A generic numerator can be expressed as a combination of (products of) denominators, multiplied by polynomial coefficients, which correspond to the residues at the multiple cuts of the diagrams. The multiple-cut conditions, applied to the loopmomenta, can be viewed as projectors isolating each residue.

In Ref. [10] a new method for the integrand reduction of one-loop amplitudes has been proposed. It exploits the knowledge of the analytic structure of the integrand on the multiple cuts in order to simplify the evaluation of the coefficients of the MI's by performing a Laurent expansion with respect to the variables which are not constrained by the cut conditions. The algorithm has been implemented in the semi-numerical $\mathrm{C}++$ library NINJA, which showed significant improvements both in performance and stability with respect to the original integrand-reduction approach. The library has recently been used for the computation of NLO QCD corrections to Higgs boson production in association with a top quark pair and a jet [13].

In Refs. [7, 8] the determination of the residues at the multiple cuts has been formulated as a problem of multivariate polynomial division, and solved using algebraic geometry techniques. These techniques allowed one to prove that the integrand decomposition is valid and applicable at any order in perturbation theory.

Within this framework, the residues of unitarity cuts can be interpreted as classes of an equivalence relation which identifies polynomials whose difference can be written as a combination of cut loop propagators. These residues, within what we call the fit-on-the-cut approach, can indeed be found by evaluating the integrand on values of the loop momenta where these denominators are put on-shell, as traditionally done in the one-loop case. The most general form of the residue, which only depends on the topology of the diagram, can be found as the most general remainder of the multivariate polynomial division modulo the Gröbner basis of the ideal generated by the cut denominators. The polynomial division also allows to carry out the integrand decomposition with purely algebraic operations, within what we call the divide-and-conquer approach [9]. In this case, the decomposition of the amplitude is obtained by successive polynomial divisions, which at each step generate the actual residues. In this way, the decomposition of any integrand is obtained analytically, with a finite number of algebraic operations. Therefore we may consider the latter a more general method for the integrand decomposition of loop integrals.

\section{Integrand reduction formula}

An arbitrary $\ell$-loop graph represents a $d$-dimensional integral of the form

$$
\int d^{d} \bar{q}_{1} \cdots d^{d} \bar{q}_{\ell} \underbrace{\mathscr{I}_{i_{1} \cdots i_{1} \ldots i_{n} \cdots i_{n}}}_{a_{1}}, \quad \underbrace{}_{a_{n}}, \quad \underbrace{}_{a_{1} \cdots i_{1} \cdots \underbrace{}_{a_{n}} \cdots i_{n}} \equiv \frac{\mathscr{N}_{i_{1} \cdots i_{1} \cdots i_{n} \cdots i_{n}}}{D_{i_{1}}^{a_{1}} \cdots D_{i_{n}}^{a_{n}}},
$$


where $i_{1}, \ldots, i_{n}$ are distinct indices. The numerator $\mathscr{N}$ and the denominators $D_{i}$ are polynomials in a set of coordinates $\mathbf{z}$. Let $P[\mathbf{z}]$ be the ring of all polynomials in such coordinates. Every set of indices $\left\{i_{1}, \ldots, i_{n}\right\}$ defines the ideal

$$
\mathscr{J}_{a_{a_{1}}}^{\mathscr{i}_{1} \cdots i_{1} \ldots i_{n_{n} \cdots i_{n}}}=\mathscr{J}_{a_{i_{1} i_{2} \cdots i_{n}}} \equiv\left\langle D_{i_{1}}, \ldots, D_{i_{n}}\right\rangle=\left\{\sum_{k=1}^{n} h_{k}(\mathbf{z}) D_{i_{k}}(\mathbf{z}): h_{k}(\mathbf{z}) \in P[\mathbf{z}]\right\} \text {. }
$$

The goal of the integrand reduction is to find a decomposition of the integrand of the form

$$
\mathscr{I}_{i_{i_{1} \cdots i_{1}} \ldots i_{n_{1} \cdots i_{n}}} \underbrace{}_{a_{n}} \equiv \frac{\mathscr{N}_{i_{1} \cdots i_{1} \cdots i_{n} \cdots i_{n}}}{D_{i_{1}}^{a_{1} \cdots D_{i_{n}}^{a_{n}}}}=\sum_{b_{1}=0}^{a_{1}} \ldots \sum_{b_{n}=0}^{a_{n}} \frac{1}{D_{i_{1}}^{b_{1}} \cdots D_{i_{n}}^{b_{n}}} \underbrace{\Delta_{i_{1} \cdots i_{1}} \cdots \underbrace{i_{n} \cdots i_{n}}_{b_{n}}}_{b_{1}}
$$

where the residues $\Delta_{i_{1} \cdots i_{1} \cdots i_{n} \cdots i_{n}}$ are irreducible polynomials, i.e. polynomials which contain no contribution belonging in the corresponding ideal $\mathscr{J}_{i_{1} \cdots i_{1} \cdots i_{n} \cdots i_{n}}$.

The numerator $\mathscr{N}$ of the integrand can be decomposed by performing the multivariate polynomial division modulo a Gröbner basis $\mathscr{G}_{i_{1} \cdots i_{n}}$ of $\mathscr{J}_{i_{1} \cdots i_{n}}$ as

$$
\mathscr{N}_{i_{1} \cdots i_{1} \cdots i_{n} \cdots i_{n}}=\Gamma_{i_{1} \cdots i_{1} \cdots i_{n} \cdots i_{n}}+\Delta_{i_{1} \cdots i_{1} \cdots i_{n} \cdots i_{n}}
$$

in terms of a quotient $\Gamma_{i_{1} \cdots i_{1} \cdots i_{n} \cdots i_{n}}$ and the remainder $\Delta_{i_{1} \cdots i_{1} \cdots i_{n} \cdots i_{n}}$. The properties of Gröbner bases ensure that the remainder is irreducible, therefore it is identified with the residue of the multiple cut $D_{i_{1}}^{a_{1}}=\cdots=D_{i_{n}}^{a_{n}}=0$, as suggested by the notation. The quotient $\Gamma$, instead, belongs to the ideal $\mathscr{J}$, thus it can be written as

$$
\Gamma_{i_{1} \cdots i_{1} \cdots i_{n} \cdots i_{n}}=\sum_{k=1}^{n} \underbrace{\mathscr{N}_{i_{1} \cdots i_{1} \ldots i_{k} \cdots i_{k} \ldots i_{n} \cdots i_{n}}}_{a_{1}} \underbrace{}_{a_{k}-1} D_{i_{k}} .
$$

Substituting Eqs. (2.3) and (2.4) in Eq. (2.1), we obtain the recursive formula [8,9]

$$
\mathscr{I}_{i_{i_{1} \cdots i_{1}} \cdots \underbrace{i_{n} \cdots i_{n}}_{a_{n}}}=\sum_{k=1}^{n} \underbrace{\mathscr{I}_{i_{1} \cdots i_{1} \ldots i_{k} \cdots i_{k} \cdots i_{k} \cdots \cdots i_{n}}}_{a_{1}} \underbrace{\Delta_{a_{n}}}_{a_{k}-1}+\frac{\Delta_{i_{1} \cdots i_{1} \cdots i_{n} \cdots i_{n}}}{D_{i_{1}}^{a_{1}} \cdots D_{i_{n}}^{a_{k}}} .
$$

Eq. (2.5) expresses a given integrand in terms of an irreducible residue sitting over its denominators and a sum integrands with fewer denominators. Hence, the recursive application of this formula ultimately yields the full decomposition of any integrand in terms of irreducible residues and denominators, as in Eq. (2).

In Ref. [8], we applied the recursive formula in Eq. (2.5) to the most general one-loop integrand. This allowed to easily derive the well know OPP decomposition for dimensionallyregulated one-loop amplitudes [3,4], as well as its higher-rank generalization for effective and nonrenormalizable theories [10], where every integrand is expressed in terms of irreducible residues with five or less denominators. Such parametrization of higher-rank residues has been used in the computation of NLO QCD corrections to Higgs boson production plus two [14] and three jets [15] in gluon fusion, in the infinite top-mass approximation.

Within the divide-and-conquer approach, the application of the integrand reduction formula allows to perform the decomposition algebraically. The latter consists in the application of Eq. (2.5) 


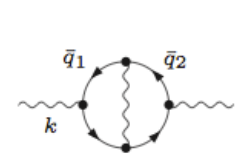

$(a)$

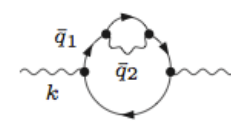

(b)

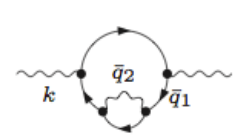

$(c)$

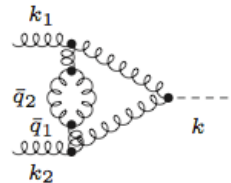

(d)

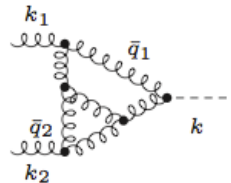

(e)

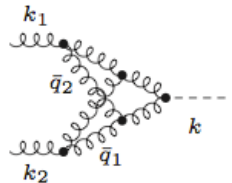

$(f)$

Figure 1: In (a), (b) and (c) are depicted the diagrams leading to the two-loop QED corrections to the photon self energy. In (d), (e) and (f) we show three two-loop diagrams entering the QCD corrections to $g g \rightarrow H$ in the heavy top mass approximation.

to the numerator of a given graph, which is used to obtain the decomposition of the amplitude by successive polynomial divisions. At each step, the remainders of the divisions are identified with the residues of the corresponding set of denominators, while the quotients become the numerators of the lower-point integrands appearing on the r.h.s. of the formula, allowing thus to iterate the procedure. In this way, the decomposition of any integrand is obtained analytically, with a finite number of algebraic operations, without requiring the knowledge of the varieties of solutions of the multiple cuts, nor the one of the parametric form of the residues.

This algorithm has been applied in [9] to the two-loop diagrams contributing to the NNLO corrections to the photon self-energy in massive QED as well as to two-loop diagrams for Higgs boson production via gluon fusion at NNLO in the heavy top limit (see Fig. 1). Despite their simplicity, these examples show the broadness of applicability of the method which is not affected by the presence of massive propagators, non planar diagrams, higher powers of loop denominators or higher-rank contributions in the numerator.

\section{Integrand-Reduction via Laurent Expansion with GOSAM and NINJA}

An improved version of integrand-reduction method for one-loop amplitudes was presented in [10], elaborating on the the techniques proposed in $[16,17]$. Whenever the analytic dependence of the integrand on the loop momentum is known, this method allows to extract the unknown coefficients of the residues by performing a Laurent expansion.

The on-shell solutions of a generic cut are parametrized by those components of the loop momentum which are not completely determined by the multiple-cut conditions. Within the original integrand reduction algorithm [18-20], the fit of the unknown coefficients which parametrize the residue of a multiple cut is performed by sampling the numerator on a finite subset of the corresponding on-shell solutions and subtracting from the integrand all the non-vanishing contributions coming from higher-point residues. This yields a linear system of equations for the unknown coefficients.

This algorithm can be simplified by exploiting the knowledge of the asymptotic behaviour of the integrand on the multiple cuts. Indeed, by performing a Laurent expansion with respect to one of the free parameters which appear in the on-shell solutions, both the integrand and the higherpoint subtractions exhibit the same polynomial behavior as the residue. Hence, the unknown coefficients of the polynomial residues can be simply identified with the ones of the Laurent expansion 


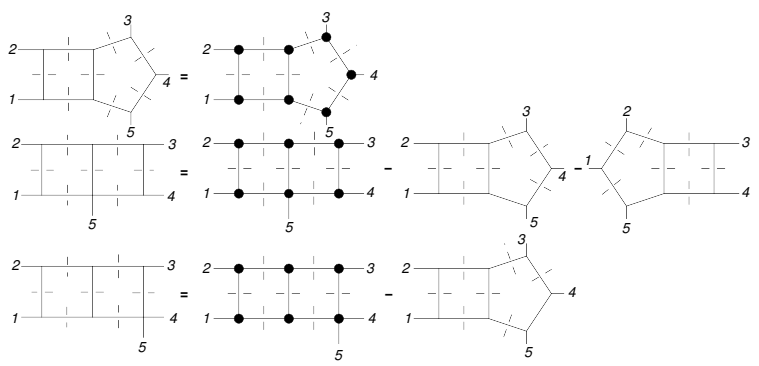

Figure 2: The eight- and sevenpole unitarity cut of the pentabox graph, which can be used to determine the corresponding residues.

of the integrand, corrected by the contributions coming from higher-point residues. In other words, with this approach the system of equations for the coefficients becomes diagonal and the subtractions of higher-point contributions can be implemented as corrections at the coefficient level which replace the subtractions at the integrand level of the original algorithm. The parametric form of this corrections can be computed once and for all, in terms of a subset of the higher-point coefficients. This also allows to significantly reduce the number of coefficients entering in each subtraction. For instance, box and pentagons do not affect at all the computation of lower-points residues.

This reduction algorithm has been implemented in the semi-numerical C++ library NINJA, which has been interfaced with the package GoSAM [11,12] for automated one-loop computations. Since the integrand of a loop amplitude is a rational function, its semi-numerical Laurent expansion has been implemented as a simplified polynomial division between the numerator and the denominators. The simplified fit of the coefficients and the subtractions at coefficient level make the algorithm implemented in NINJA significantly lighter, faster and more stable than the original. Moreover the extension of this approach to higher-rank integrands is particularly easy, and indeed the library can also be used for the reduction of higher-rank integrands where the rank of a numerator can exceed the number of denominators by one.

The first new phenomenological application of NINJA has been the computation of NLO QCD corrections to Higgs boson production in association with a top quark pair and a jet [13]. The possibility of exploiting the improved stability of the new algorithm has been especially important for the computation of the corresponding six-point virtual amplitude, given the presence of two mass scales as well as massive loop propagators which make traditional integrand reduction algorithms numerically unstable. Indeed, for the highly non-trivial process under consideration, only a number of phase-space points of the order of one per mill were detected as unstable. All these points have been recovered using the tensorial reduction provided by GoLEM95 [21,22], thus avoiding the necessity of higher precision routines, which are extremely time consuming.

\section{Application to Two-Loop Scattering Amplitudes in $\mathscr{N}=4 \mathrm{SYM}$}

The integrand reduction within the fit-on-the-cut approach has been combined with the colorkinematic duality [25] to construct the two-loop five-point amplitude for $\mathscr{N}=4$ super Yang-Mills (sYM) [26]. 


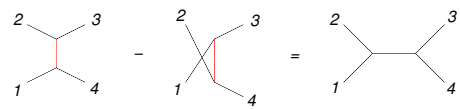

Figure 3: Graph representation of the Jacobi Identity at four points

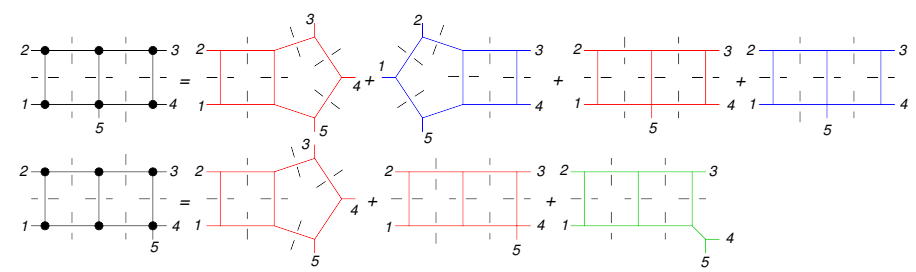

Figure 4: Each seven-pole unitarity cut is split into two contributions as indicated by the colors. The green diagram represents the new seven-denominator topology arising from the BCJ equations.

The input for the integrand decomposition are, on the one-side, the products of the trees to be sewn along the multiple-cuts, and on the others the parametric residues and solutions of the multiple-cuts. The former can be computed by adopting the super-amplitude formalism [28], while the latter were classified in [27].

The reduction of the amplitude, in this case, begins from the eight-pole cuts, which is a maximum-cut [8], and terminates after determining the residues at the sevenpole-cuts. The absence of lower cuts is compatible with the properties that $\mathscr{N}=4 \mathrm{sYM}$ integrands are linear in the loop-momenta. Representative steps of the reduction are given in Fig. 2.

Once the reduction is completed, one can equivalenty construct a numerator function for the parent eight-denominator topologies, which capture the whole structure of the scattering amplitude. These numerators can be rearranged in a color-kinematic dual form by imposing additional constraints, referred to as BCJ equations, derived from the the kinematical equivalent of the Jacobi Identity portrayed in Fig. 3. BCJ identities, beyond one-loop, imply a relation between the integrands of the planar and of the non-planar topologies. The color-kinematic dual numerator for the eight-denominator planar diagram is represented in the first line of Fig.5. The key equations for the determination of the new numerators of the planar diagrams are depicted in Figs. 4, where one may notice the rising of a seven-denominator diagram, whose identification was not needed in the unitarity decomposition. In order to disentangle the contributions to the sevenpole cuts we use the BCJ equations which only involve the planar topologies displayed in the last two lines of Fig. 5. The obtained results are in agreement with [29].

\section{Conclusions}

We described a coherent framework for the decomposition of Feynman integrals, which can be applied at any loop order, regardless of the complexity of the integrand, the number of external legs or the presence of higher powers of loop denominators. This framework allows to easily derive well known results at one-loop order and extend them to higher loops.

In the one-loop case, we showed how the knowledge of the analytic structure of the integrands on the multiple cuts, and in particular their asymptotic behaviour on the on-shell solutions, can be used to improve the analytic reduction with the Laurent expansion method. Its implementation 


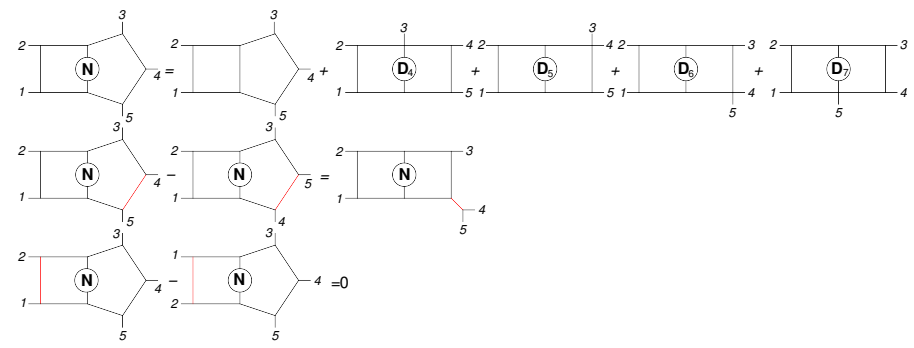

Figure 5: The first line shows the decomposition of the numerator $N$ of the planar topology, in terms of an eight-pole-cut residue and the seven-pole-cut residues multiplied by the corresponding denominators $D_{i}$. The last two lines show the BCJ equations which can be used to disentangle the sevenpole unitarity cuts.

in the C++ library NINJA provided a considerable gain in the speed and in the stability of the reduction.

At higher loops, we showed the application of the integrand decomposition both within the fit-on-the-cut approach and the divide-and-conquer approach. With the former the residues are evaluated on the corresponding multiple cuts and we showed an application in $\mathscr{N}=4$ SYM using the super-amplitude formalism. Using the latter we can instead perform the full decomposition with purely algebraic operations on the numerator and the set of denominators of a given integrand. We applied it to simple examples, some of which cannot be addressed with other unitarity-based and integrand-reduction methods, due to the presence of higher powers of loop denominators in the integrands. The algebraic reduction also allows to avoid the determination of the algebraic variety defined by the on-shell solutions, which is a non-trivial task especially for those multiple cuts which leave several unconstrained variables.

\section{Acknowledgments}

The work of H.v.D., G.L., P.M., T.P. and U.S. was supported by the Alexander von Humboldt Foundation, in the framework of the Sofja Kovaleskaja Award Project "Advanced Mathematical Methods for Particle Physics", endowed by the German Federal Ministry of Education and Research. G.O. was supported in part by the National Science Foundation under Grant PHY-1068550.

\section{References}

[1] Z. Bern, L. J. Dixon, D. C. Dunbar and D. A. Kosower, Nucl. Phys. B 425 (1994) 217.

[2] F. Cachazo, P. Svrcek and E. Witten, JHEP 0409 (2004) 006.

[3] G. Ossola, C. G. Papadopoulos and R. Pittau, Nucl. Phys. B 763 (2007) 147;

G. Ossola, C. G. Papadopoulos and R. Pittau, JHEP 0707 (2007) 085;

G. Ossola, C. G. Papadopoulos and R. Pittau, JHEP 0805 (2008) 004.

[4] R. K. Ellis, W. T. Giele and Z. Kunszt, JHEP 0803 (2008) 003.

R. K. Ellis, W. T. Giele, Z. Kunszt and K. Melnikov, Nucl. Phys. B 822 (2009) 270;

W. T. Giele, Z. Kunszt and K. Melnikov, JHEP 0804 (2008) 049.

[5] P. Mastrolia and G. Ossola, JHEP 1111, 014 (2011). 
[6] S. Badger, H. Frellesvig and Y. Zhang, JHEP 1204 (2012) 055.

[7] Y. Zhang, JHEP 1209 (2012) 042.

[8] P. Mastrolia, E. Mirabella, G. Ossola and T. Peraro, Phys. Lett. B 718 (2012) 173.

[9] P. Mastrolia, E. Mirabella, G. Ossola and T. Peraro, arXiv:1307.5832 [hep-ph].

[10] P. Mastrolia, E. Mirabella and T. Peraro, JHEP 1206 (2012) 095.

[11] G. Cullen, N. Greiner, G. Heinrich, G. Luisoni, P. Mastrolia, G. Ossola, T. Reiter and F. Tramontano, Eur. Phys. J. C 72 (2012) 1889.

[12] G. Ossola, presentation at EPS-HEP 2013, in these proceedings.

[13] H. van Deurzen, G. Luisoni, P. Mastrolia, E. Mirabella, G. Ossola and T. Peraro, arXiv:1307.8437 [hep-ph].

[14] H. van Deurzen, N. Greiner, G. Luisoni, P. Mastrolia, E. Mirabella, G. Ossola, T. Peraro and J. F. von Soden-Fraunhofen et al., Phys. Lett. B 721 (2013) 74.

[15] G. Cullen, H. van Deurzen, N. Greiner, G. Luisoni, P. Mastrolia, E. Mirabella, G. Ossola and T. Peraro et al., Phys. Rev. Lett. 111 (2013) 131801.

[16] D. Forde, Phys. Rev. D 75 (2007) 125019.

[17] S. D. Badger, JHEP 0901 (2009) 049.

[18] G. Ossola, C. G. Papadopoulos and R. Pittau, JHEP 0803 (2008) 042.

[19] P. Mastrolia, G. Ossola, C. G. Papadopoulos and R. Pittau, JHEP 0806 (2008) 030.

[20] P. Mastrolia, G. Ossola, T. Reiter and F. Tramontano, JHEP 1008 (2010) 080.

[21] T. Binoth et al., Comput. Phys. Commun. 180 (2009) 2317.

[22] G. Cullen, J. P. .Guillet, G. Heinrich, T. Kleinschmidt, E. Pilon, T. Reiter and M. Rodgers, Comput. Phys. Commun. 182 (2011) 2276.

[23] R. Britto, F. Cachazo and B. Feng, Nucl. Phys. B 715 (2005) 499.

[24] R. Britto, F. Cachazo and B. Feng, Nucl. Phys. B 725 (2005) 275.

[25] Z. Bern, J. J. M. Carrasco and H. Johansson, Phys. Rev. D 78 (2008) 085011.

[26] U. Schubert, Diplomarbeit (2013) .

[27] P. Mastrolia, E. Mirabella, G. Ossola and T. Peraro, Phys. Rev. D 87 (2013) 085026.

[28] J. M. Drummond, J. Henn, G. P. Korchemsky and E. Sokatchev, Nucl. Phys. B 869 (2013) 452.

[29] J. J. .Carrasco and H. Johansson, Phys. Rev. D 85 (2012) 025006. 